Int. J. Dev. Biol. 49: 375-389 (2005)

doi: $10.1387 / \mathrm{ijdb} .041910 \mathrm{cw}$

Special Contribution

\title{
Limb developmental stages of the newt Notophthalmus viridescens
}

\author{
CHRISTINE J. WONG* and RICHARD A. LIVERSAGE \\ Ramsay Wright Zoological Laboratories, University of Toronto, \\ Toronto, Ontario, Canada
}

\section{Foreword}

The current study consists of a detailed description of normal larval forelimb and hindlimb development in the newt, Notophthalmus viridescens. This is the first comprehensive record of staging limb development in this urodele amphibian species, augmenting Fankhauser's work which was published in fragments 60 years ago. Larvae were obtained by natural spawning, without hormone injections and while experimental conditions such as temperature and light cycles were kept constant $\left(20^{\circ} \mathrm{C}\right.$ and 12L/12D), larval sizes have been added here merely as guidelines as development is influenced by a number of extrinsic factors. Care has been taken to indicate similarities to either the Harrison or Glücksohn stages, although this relates mainly to their morphological descriptions of the limbs. Observations from limb use are also mentioned in the text where pertinent to the description of development.

KEY WORDS: Notophthalmus viridescens, urodele, development, forelimbs, hindlimbs

\section{Introduction}

The adult red spotted newt Notophthalmus viridescens has commonly been used in North America for regeneration studies due to their ability to regenerate their appendages as well as

structures of their eye, spinal cord and heart muscle. More recently, larval newts have been used to compare known processes of forelimb development with those of adult forelimb regeneration (Cadinouche et al., 1999; Vlaskalin et al., 2004) to better understand these processes. While urodeles have fallen out of favour for embryological studies, what has become evident is that some developmental processes are not conserved in this amphibian. For example, urodeles fail to form a thickened apical ectodermal ridge (Sturdee \& Connock, 1975), which might explain the boundary-independent pattern of fringe expression observed during $N$. viridescens limb development (Cadinouche et al., 1999).

A detailed description of normal limb development in $N$. viridescens, however, is absent from the literature. While Fankhauser $(1945,1967)$ describes normal development for $N$. viridescens, these reports are somewhat fragmentary and recent literature has utilized either Glücksohn's (1931) Triton, Harrison's (1969) Ambystoma or Shi and Boucaut's (1995) Pleurodeles publications to stage $N$. viridescens limb development. There are, however, limitations in using these earlier works. For example, Harrison's published work does not encompass the entire course of limb morphogenesis, ending during the early stages of hindlimb development and before forelimb development has been com-

Abbreviations used in this paper: DI, digit I stage; e2D, early two digit stage; $\mathrm{h}$, average head length; $\mathrm{L}$, average total length; $\mathrm{t}$, average tail length. *Address correspondence to: Dr. Christine J. Wong. Samuel Lunenfeld Research Institute, Mount Sinai Hospital, 600 University Ave., Rm. 876, Toronto, ON,
Canada, M5G 1X5. Fax:+1-416-946-5536. e-mail: cwong@mshri.on.ca 
pleted. It is also important to remain within the same genera of urodeles for comparative staging of limb development. Thus, comparing $N$. viridescens development with Glücksohn's Triton species is more pertinent than with Harrison's Ambystoma species (Duellman \& Trueb, 1986).

There are also concerns with the work of Glücksohn (1931), as the publication describes two European species of Triton larvae. Therefore, care must be taken to specify which species is being referred to, inasmuch as development is occasionally more advanced in $T$. taeniatus than for the similarly numbered stages of T. cristatus larvae. Another concern with Glücksohn's work is that having observed abnormal changes in the development patterns of larvae contained in her temperature-controlled incubators, she chose to raise larvae at room temperature. While the room temperature averaged $19-21^{\circ} \mathrm{C}$, larvae were exposed to extremes of $16.5^{\circ} \mathrm{C}$ and $25^{\circ} \mathrm{C}$. Glücksohn reasoned that her larvae would be experiencing such temperature extremes in their natural environment. However, her latter conditions would be difficult to re-create in a laboratory setting.

The current research provides a detailed description of limb development in the newt by observing and recording major morphological changes. This method was accomplished in a manner similar to the detailing of normal stages during epimorphic forelimb regeneration in adult $N$. viridescens (Iten \& Bryant, 1973), with stages more descriptive-based than numericallybased staging, inasmuch as limb development lends itself well to this method. Although the use of numbers to essentially count up the progression of development has historically been utilized for many species, both Gallien and Durocher (1957) and Knight (1937) inadvertently chronicle the problems inherent in the numerical method of identification. Firstly, there has been a disparity among investigators in choosing the initiation time of development. Thus, while some researchers begin staging at the uncleaved egg, others consider the first cell cleavage appropriate for the designation of stage 1 . Secondly, how are the numbers of stages determined? While investigators use developmental changes to external morphology as their criteria, some note relatively minute changes, while others consider these added stages superfluous. Utilizing stained skeletal elements as the criterion, we demonstrate that the use of relatively small morphological changes to designate a new stage is often inconsequential, even though an external change is evident. The fluidity of development is such that once the larval stage has been attained, much of its cellular and tissue differentiation continues long after the major changes in morphology are observed.

\section{Forelimb development}

\section{Anterior limb field: $L$ (average total length) $=5.8 \mathrm{~mm}, t$ (average} tail length) $=1.2 \mathrm{~mm}, h$ (average head length) $=1 \mathrm{~mm}$

Lateral local distension of the body wall at the level of the developing gill region indicates the anterior limb field.

Bilateral gill sprouts and more rostral balancer buds are located caudal to the eye and are similar in length. Protruding eye structures are obvious, but devoid of black pigment. Pigmentation of the skin appears dorsally and laterally along the length of the embryo, as well as in the pericardial sac. A small dorsal fin extends from the mid-body caudally, while a larger ventral tail fin extends from the caudal margin of the yolk mass further posterior. A mid-ventral blood vessel is observed ventrally along the internal area of the yolk mass, containing blood, with a bolus of blood observed to move through the beating heart.

\section{Bud stage ( $L=7.2 \mathrm{~mm}, t=2.1 \mathrm{~mm}, h=1 \mathrm{~mm}$ )}

Although originally growing out from the lateral body wall perpendicular to the body axis, at this stage the forelimb buds have become caudally-oriented with a dome-shaped distal tip. In dorsal view, the pre-axial edge of the bud is greater in length than the postaxial edge. In lateral view, the forelimb bud is dorsally oriented. Pigmentation appears on the forelimb, but limited dorsally. The limb bud is composed of a mesenchymal cell mass, contained in a sleeve of epithelium.

The rami of external gills have developed sufficiently to extend beyond the length of the limb buds and fimbriae have become apparent along the ventral edges of the gills. Balancers are similar to the external gills in size. Both the dorsal and ventral fins have grown larger. Pigmentation is developing within the eye, although limited in the dorsal eye region. The mouth of the larva is located ventrally on the head, slightly caudal to and beneath the rounded snout.

\section{Peg stage ( $L=7.9 \mathrm{~mm}, t=2.9 \mathrm{~mm}, h=1.1 \mathrm{~mm}$ )}

The peg stage is so described, because in dorsal view, the preand postaxial edges are parallel. In lateral view, however, the dorsal and ventral edges taper distally, resulting in a cone-shaped forelimb. The limb now points dorso-caudally in lateral view. The peg appears in two forms, first as a domed distal tip then as a more transient squared tip, the latter occurring immediately prior to digit outgrowth. In dorsal view, the forelimb is held at approximately a $30^{\circ}$ angle from the body.

Embryos emerge from their egg capsule at this stage. The balancers are longer than the external gills and the distal tips of the balancers extend ventrally to anchor the embryo as well as balance it in an upright position. The pericardial sac is heavily pigmented and blood flows through the gills in synchrony with the heart beat. Black pigmentation has encroached within most of the eye, with golden flecking beginning to overlay the pigmentation. Each elongated rami of the external gills has two rows of fimbraie. The opercula are located ventrally on the head, extending to the midline. The cranial portion of the yolk mass has begun to change colour and becomes opaque in the region of the presumptive liver.

\section{e2D stage ( $L=8.9 \mathrm{~mm}, t=3.5 \mathrm{~mm}, h=1.4 \mathrm{~mm}$ )}

At this early (e) two digit (2D) stage, digits evolve as small knobs (as height $=$ width) developing from the lateral edges of the distal region of the forelimb, with a distinct interdigital depressed notch forming medially. Digits at this stage are of equal length and shape. In dorsal view, the pre- and postaxial edges are still parallel along the length of the limb, nevertheless the edges of the limbs are curvilinear. In lateral view, the limb tapers to a flattened distal tip, the proximal part of the limb remaining thickened. From this stage onward, the forelimb begins to rotate, which results in the digits changing from being aligned horizontally to aligning vertically. As such, the palm of this appendage rotates to finally face medially. Little or no Alcian staining (Fig. 1A) can be seen along the length of the forelimb during this stage, although a cartilage nodule forms proximally in the shoulder region of the forelimb.

The mouth of the larva is now oriented more rostrally on the head 
and lies at the rostral border of the eye.

\section{$2 D(L=9.6 \mathrm{~mm}, t=3.9 \mathrm{~mm}, h=1.7 \mathrm{~mm})$}

By the two digit (2D) stage, the forelimb has completed its rotation with the result both digits are aligned vertically, with the palm directed medially and open towards the body. The proximal part of the forelimb is thinner in lateral view than the hand. Digits are now triangular in shape, the base of which adjoins the hand while the apex forms the distal digit tip. Digit one (D1) is smaller than the more dorsally-situated digit two (D2). The apex of the caudallyoriented D2 lies medially from the distal tip of the forelimb while D1 points ventrally. The limb now flattens distally in dorsal view while the long edges of the forelimb remain parallel. The limb becomes curvilinear along its length.

The liver is observed in ventral view as an organ lying immediately cranial to the yolk sac, but caudal to the heart.

\section{e3D ( $L=9.7 \mathrm{~mm}, t=4.2 \mathrm{~mm}, h=1.8 \mathrm{~mm})$}

At the early three digit (e3D) stage, the entire hand can readily be observed in dorsal view beyond the end of the gills and it is broader than the rest of the forelimb. While D2 remains larger and triangular in shape, D1 is elongating. Together with the balancers, the animal uses D1 for balance. A flattening forms on the developing hand, angling ventrally from the dorsal edge of the forelimb to the base of D2. It is from this region that digit three (D3) will form. Ventral angling at the dorsal midpoint of the edge of the limb suggests a demarcation between the stylopodium and the zeugopodium. By the e3D stage, Alcian-stained cartilage, in the thin proximal part of the forelimb, has condensed into a rod-shaped structure namely, the presumptive humerus (Fig. 1B). Cartilage in the shoulder region is distinct from the proximal part of the humerus rod, indicative of an articulation surface. Procartilage aggregates appear in the zeugopodium. Muscle is observed to extend into the proximal portion of the limb from the shoulder region.

The blood vessel lying mid-ventrally in the yolk sac enters the liver and blood disperses from this point, circulating through the liver before entering mid-ventrally into the heart. The cranial portion of the yolk mass takes on an irregular shape with minor constrictions observed along the edges of the yolk sac.

\section{DI stage ( $L=10.8 \mathrm{~mm}, t=4.9 \mathrm{~mm}, h=2 \mathrm{~mm}$ )}

At the 3 digit I (3DI) stage D3 emerges from the flattened area of the hand, developing into a knob lying along the ulnar side of the forelimb. The limb is now no longer caudally-oriented, but rather, held bent at the elbow at $90^{\circ}$ angle to the body axis. As such, the larva uses D1 (and eventually D2) to brace itself upright as the hand has developed and rotated cranially. D1 and D2 have elongated, although D2 remains the longer digit, aligned medially on the forelimb. Alcian-stained cartilage is observed along the entire length of the forelimb (Fig. 1C). While the proximal portion has formed a humerus, cartilage is beginning to condense into a separate ulna and radius. Although well-defined cartilage rods are discernible in D1 and D2, no cartilage is observed within the knobshaped D3. Diffuse Alcian staining also occurs in the distal zeugopodial and presumptive carpal areas.

The gut begins to form as the cranial portion of the yolk mass takes on a distinct tubular shape. Larvae begin to eat at this stage. The head develops a more rectangular shape and the balancers become thin and often curl distally or break.

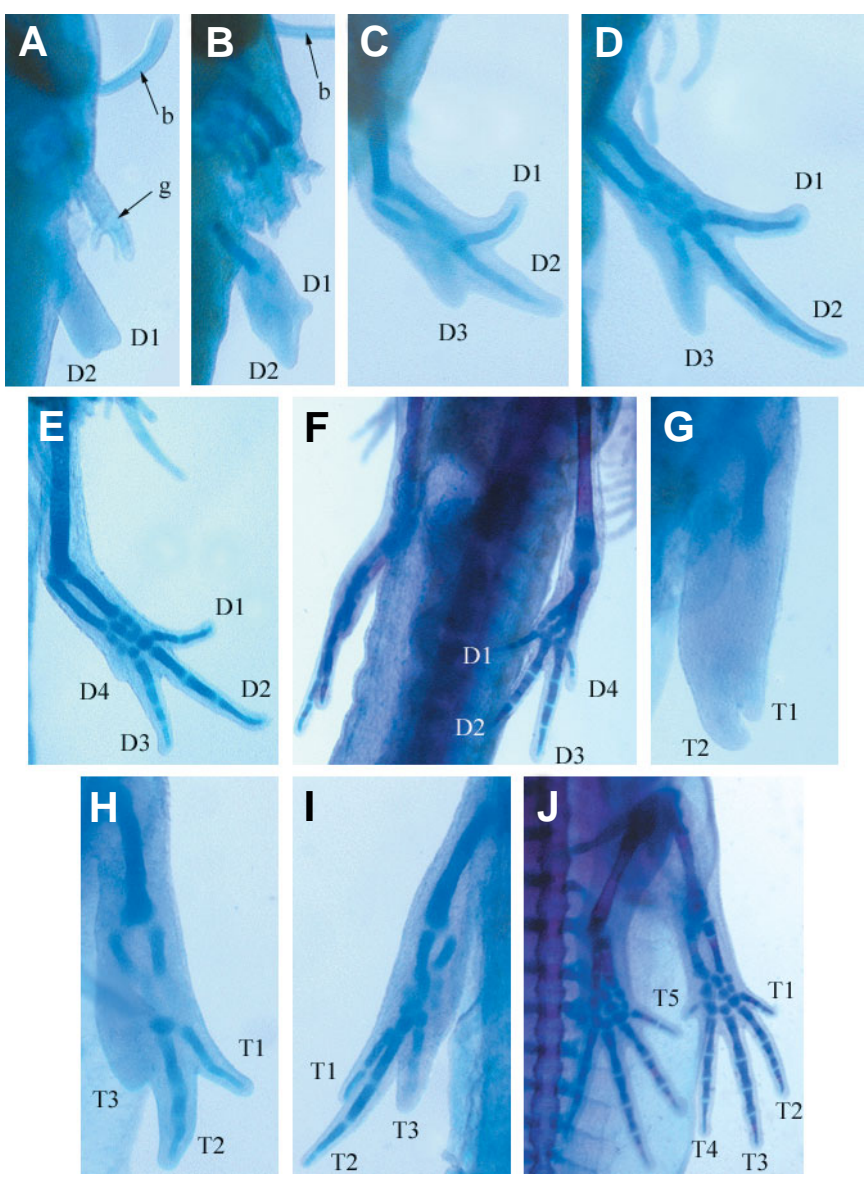

Fig. 1. Differentiation of cartilage in the developing fore- and hindlimbs. (A) Early two digit forelimb stage. The caudal two external gill filaments (g) have been removed, while a balancer (b) can be seen rostrally. No Alcian Blue-stained cartilage is observed along the length of the forelimb nor in digits 1 (D1) and 2 (D2) (x20). (B) Early three digit forelimb stage. All external gill filaments have been removed, but the rostral balancer (b) is still present. An Alcian Blue-stained cartilage rod has formed within the forelimb proximally, with diffusely stained material throughout the forearm (x28). (C) Three digit I forelimb stage. Proximal regions of the radius and ulna articulate with the humerus, with diffuse Alcian Blue staining in the remainder of the forearm. Rods of cartilage support D1 and D2 while no Alcian Blue staining extends into digit 3 (D3) (x34). (D) Three digit II forelimb stage. Carpals form adjacent to the distal ends of the radius and ulna while cartilage differentiation continues in D1, D2 and D3 (x40). (E) Four digit forelimb stage. Distinct cartilages have formed in the limb although no cartilage has formed in the knob shaped digit 4 (D4) (x30). (F) Four digit forelimb stage. Alazarin Red-stained bone has formed in the humerus and Alcian Blue-stained cartilages appear in all four phalanges (x32). (G) Two toe hindlimb stage. Cartilage condenses proximally into a rod-shaped femur while the distal hindlimb, including toes 1 (T1) and 2 (T2), lack cartilage staining (x65). (H) Three toe hindlimb stage. Cartilage is observed in T1 and T2 and also forms the proximal ends of the tibia and fibula, as well as the femur, although distinct tarsal elements have not yet formed and cartilage staining is absent from toe 3 (T3) (x54). (I) Three toe hindlimb stage. Cartilage differentiates into distinct elements along the length of the developing limb (x46). (J) Five toe hindlimb stage. Alazarin Red-stained bone is observed in the shaft of the femur while all other toes (T1-T5) contain distinct cartilages $(\times 15)$. 


\section{DIl stage ( $L=12.5 \mathrm{~mm}, t=5.6 \mathrm{~mm}, h=2 \mathrm{~mm}$ )}

At the 3 digit II (3DII) stage, elongation of D1, D2 and D3 continues, with all three becoming digit-like in appearance. These digits touch the underlying substrate, palm facing ventrally. D2 remains the longest digit, with the elongating D3 approaching the same length as D1. In dorsal view, the stylo- and zeugopodia are of similar thickness. By this stage, a well-formed glenoid cavity in the shoulder girdle articulates with the humerus. The entire length of the humerus, as well as the proximal components of the radius and ulna have formed. Cartilage aggregates at the distal zeugopodium. The carpus cartilage has not yet separated into these distinct cartilage elements (Fig. 1D). D1 and D2 consist of unseparated rods of cartilage, although an accumulation of cells within D2 suggests the location of a presumptive metacarpal joint. A cartilage aggregate extends into D3. This digit is composed distally of a collection of aligned mesenchymal cells.

The entire gut becomes tubular within the peritoneal cavity. The cranial portion begins to twist into loops while the caudal portion remains linear, except for slight constrictions of the gut wall along it length, presumably in advance of the caudal progression of gut development. Some yolk material is still observed along the caudal portion of the gut, but it is absent from the cranial region of the gut. The snout has become more tapered rostrally, with the head wider at the gill region. As well, larva begin to show movement of the head from side to side.

\section{D stage ( $L=13 \mathrm{~mm}, t=6.2 \mathrm{~mm}, h=2.3 \mathrm{~mm}$ )}

By the 4 digit (4D) stage, digit four (D4) initially forms as a small hillock at a right angle to the hand and adjacent to D3. As this hillock evolves into a knob, a depression or valley forms between D3 and D4, as D4 develops to align with the other digits. By the time D4 has formed as a knob-shaped protuberance, cartilage within all forelimb elements have condensed along their length, except for the skeletal support of D4 (Fig. 1E). However, cartilage condensation of D4 takes place as it elongates and becomes a distinct digit, whereupon the normal process of ossification of forelimb cartilage elements begins (Fig. 1F). As morphogenesis progresses, D3 approaches and eventually supercedes D2, slightly, in its final length, while the final extent of D4 approaches that of D1. That is to say, D3>D2>D1>D4.

At this stage, the forelimb is used to co-ordinate a walking motion, allowing the larva to actively move forward.

\section{Hindlimb development}

Posterior limb field: $L$ (average total length) $=12.2 \mathrm{~mm}, t$ (average tail length) $=5.7 \mathrm{~mm}, \mathrm{~h}$ (average head length) $=2 \mathrm{~mm}$

The beginning of hindlimb development is variable, emerging anywhere between forelimb stages 3DII and 4D. Thickening of the body wall at the hindlimb region occurs, such that small bilateral patches are visible ventrally, immediately cranial and lateral to the cloacal aperature. These are formed by the accumulation of loosely-packed mesenchymal cells in this area, subjacent to the epithelium.

\section{Bud I stage ( $L=12.6 \mathrm{~mm}, t=5.77 \mathrm{~mm}, h=2.15 \mathrm{~mm}$ )}

As outgrowth begins, these limbs appear as small mounds at right angles to the ventral body wall, slightly cranial and lateral to the cloaca.

\section{Bud II stage ( $L=12.8 \mathrm{~mm}, t=6.1 \mathrm{~mm}, h=2.26 \mathrm{~mm}$ )}

As the limb bud forms, the preaxial length increases prior to development of the postaxial length, with the result the hindlimb bud develops in an 'L'-shape manner in ventral view. That is to say, the elongated preaxial length lies along the ventral body wall, parallel to the body axis, while the postaxial edge forms perpendicular to this caudal margin.

\section{Bud III stage ( $L=13.6 \mathrm{~mm}, t=6.5 \mathrm{~mm}, h=2.3 \mathrm{~mm}$ )}

Outgrowth begins with the formation of a postaxial edge on the developing bud. This is identified by an indentation along the caudo-medial edge of the hindlimb. Elongation occurs along the caudal and lateral parts of the limb, at an angle approximately $30^{\circ}$ from the body axis. The distal limb tip is dome-shaped at this stage, which can be seen in dorsal view.

\section{eT stage ( $L=14.3 \mathrm{~mm}, t=7.1 \mathrm{~mm}, h=2.4 \mathrm{~mm}$ )}

Lengthening of the preaxial edge at the early toe (eT) stage is greater than the postaxial edge, which results in an angular flattening of the distal tip between these asymmetrical edges. Presumably, this is in advance of distal toe formation. As a result of the caudal direction of growth of the hindlimb, the appendage extends past the cloaca and the preaxial length exhibits a proximal curvature as the limb develops and lies close to the body. While the proximal part of the limb remains essentially tubeshaped, the distal portion flattens slightly in ventral view. Dorsally, the hindlimb can be observed as a thin ridge running parallel to the body axis.

\section{$2 T$ stage ( $L=14.9 \mathrm{~mm}, t=7.3 \mathrm{~mm}, h=2.7 \mathrm{~mm}$ )}

The first two toes (2T) of this limb stage appear concomitantly, with the distal tip of the limb forming toe two (T2). While T2 develops medially along the axis of the hindlimb, toe one (T1) forms on the ventral edge of the limb. While both toes are observable in dorsal view, T2 curves away from the body. In lateral view, the dorsal edge of the hindlimb forms a ridge or 'plate' from which toe 3 (T3), toe 4 (T4) and toe 5 (T5) will arise. During the 2T stage, a cartilage nodule forms within the body wall at the presumptive pelvic girdle region. A cartilaginous aggregate condenses within the proximal portion of the limb while precartilage alignment is observed distally to form the presumptive femur (Fig. $1 \mathrm{G})$.

\section{$3 T$ stage ( $L=15.7 \mathrm{~mm}, t=7.9 \mathrm{~mm}, h=2.9 \mathrm{~mm}$ )}

Toe three (T3) of the 3T stage forms as a knob along the dorsal edge of the foot. The triangular T1 and T2 elongate, while T3 passes through a knob and then a triangular shape during this stage. In lateral view, the upper part of the limb remains the same thickness as the lower portion, although a distinct knee joint forms. As development ensues, the curvature at the mid-hindlimb will delimit the knee in dorsal view, with the upper limb portion becoming thinner than the lower. A ridge or 'plate' remains on the dorsal part of the hindlimb adjacent to T3, in the region from which T4 and T5 will form. An articulation surface, well-defined between the cartilage of the pelvic girdle and the head of the femur, is discernible by the $3 \mathrm{~T}$ stage (Fig. $1 \mathrm{H}, \mathrm{I}$ ). Muscle fibers are now immediately adjacent to the proximal femur, rendering movement. While the femur and proximal portions of both the fibula and tibia form discrete skeletal elements of cartilage, the distal tibia 
and fibula part, as well as the presumptive tarsal area, consist mainly of precartilage aggregates (Fig. $1 \mathrm{H}$ ). Cartilage rods form within $\mathrm{T} 1$ and $\mathrm{T} 2$ and an alignment of mesenchymal cells now extends from the presumptive tarsus into T3. It appears that distinct metatarsals and phalangeal elements condense in the developing foot with the outgrowth and condensation of cartilage within T3, as do distinct tarsals within the ankle of the hindlimb (Fig. 1I).

During this stage, the hindlimbs will also make contact with the underlying substrate, as did the forelimbs during the e3D stage, in order to act as balancers. This contact first occurs with T1. Movement of the limb is spasmodic initially and it is not reactive to external stimuli during this stage. However, by the end of the stage, the toes respond to tactile stimulation when the hindlimb is touched by a probe which causes the larva to turn caudally toward the affected area. Nevertheless, the foot itself is not withdrawn from the stimulus.

\section{$4 T$ stage ( $L=17 \mathrm{~mm}, t=8.6 \mathrm{~mm}, h=3 \mathrm{~mm}$ )}

At the 4 toe (4T) stage, T4 emerges as a mound at a right angle to the main foot axis. As outgrowth occurs, T4 forms a knob and a depression or valley forms between T3 and T4. As the latter develops it aligns with the other digits.

The foot rotates, to eventually allow the sole to contact the substrate, with $\mathrm{T} 2$ now used along with $\mathrm{T} 1$ to anchor and balance the animal. As foot rotation progresses, the elongated T3 will also make contact with the substrate. With this elongation, T3 will approach the length of T2 and surpass that of T1. Use of the knee in movement occurs at this stage as the animal uses the hindlimbs in a walking motion. Larva also react to tactile stimulation of the hindlimb during this stage by actually moving the appendage.

\section{$5 T$ stage ( $L=19.2 \mathrm{~mm}, t=10 \mathrm{~mm}, h=3.4 \mathrm{~mm}$ )}

At the 5 toe (5T) stage, toe 5 (T5) appears as a mound on the dorsal surface of the limb adjacent to T4 and perpendicular to the axis of the foot. Similar to T4, this mound grows into a knob, with a depression or valley forming between T4 and T5 as the latter develops and rotates medially to align with the other digits. Unlike the other toes, however, T5 does not lengthen, but rather, forms a peg-shaped digit. Elongation of T4 and the limited outgrowth of
$\mathrm{T} 5$ results in the final toe lengths of $\mathrm{T} 3>\mathrm{T} 4>\mathrm{T} 2>\mathrm{T} 1>\mathrm{T} 5$. Cartilage elements continue to emerge in T1-T4 and ossification within the hindlimb is detectable (Fig. $1 \mathrm{~J})$.

\section{References}

CADINOUCHE, M.Z.A., LIVERSAGE, R.A., MULLER, W. and TSILFIDIS, C. (1999). Molecular cloning of the Notophthalmus viridescens radical fringe cDNA and characterization of its expression during forelimb development and adult forelimb regeneration. Dev. Dyn. 214: 259-268.

DUELLMAN, W.E and TRUEB, L. (1986). Biology of Amphibians. NewYork:McGrawHill.

FANKHAUSER, G. (1945). The effects of changes in chromosomal number on amphibian development. Quart. Rev. Biol. 20:21-78.

FANKHAUSER, G. (1967). Urodeles. IN Methods in Developmental Biology, (Wilt \& Wessels, eds.), Thomas Crowell Publishers, New York, pp.85-99.

GALLIEN, L. and DUROCHER, M. (1957). Table chronologique du développement chez Pleurodeles waltlii Micah. Bull. Biol. France-Belgique 9:97-114.

GLÜCKSOHNS. (1931). Äussere entwicklung der extremitaten und stadieneinteilung der larvenperiode von Triton taeniatus leyd. und Triton cristatus laur. Wilhelm Roux Archiv. fur Entwicklungsmechanik der Organismen. 125:341-406.

HARRISON, R.G. (1969). Harrison stages and description of the normal development of the spotted salamander, Amblystoma punctatum (Linn). IN Organization of the Embryo (S. Willens, ed.) Yale University Press, pp. 44-66.

ITEN, L.E. and BRYANT, S.V. (1973). Forelimb regeneration from different levels of amputation in the newt, Notophthalmus viridescens: length, rate and stages. Wilhelm Roux Archiv. 173:263-282.

KNIGHT, F.C.E. (1937). Die entwicklung von Triton alpestris bei vershiedenen temperaturen mit normentafel. W. Roux's Archiv. f. Enticklungsmechanik. 137:461-473.

SHI, D.-L. and BOUCAUT, J.-C. (1995). The chronological development of the urodele amphibian Pleurodeles waltl (Michah). Int. J. Dev. Biol. 39: 427-441.

STURDEE, A. and CONNOCK, M. (1975). The embryonic limb bud of the urodele: morphological studies of the apex. Differentiation 3:43-49.

VLASKALIN, T., WONG, C. J. and TSILFIDIS, C. (2004). Growth and apoptosis during larval forelimb development and adult forelimb regeneration in the newt, Notophthalmus viridescens. Dev. Genes Evol. 214(9):423-431.

Received: October 2004 Reviewed by Referees: December 2004 Modified by Authors and Accepted for Publication: February 2005 



\section{Table of Limb Development Stages in Notophthalmus viridescens}




\section{Plate 1. Forelimb Stages}

Limb field. Distension of the body wall at the level of the developing gill region indicates the limb field. Gill sprouts and more rostral balancers are similar in length. Corresponds to Harrison st. 35.

Bud. Forelimb bud has a dome-shaped distal tip. Rami of external gills extend beyond the length of the limb buds. Pigmentation observed dorsally in the eye. Corresponds to both Harrison and Glücksohn st. 39.

Peg. Pre- and postaxial edges of the forelimb are parallel. Embryos emerge from egg capsule. Balancers are longer than the external gills. Blood flows through gills in synchrony with heart beat. Corresponds to Glücksohn st. 40 and is similar to Harrison st. 40 .

e2D. Digits evolve as small knobs developing along the lateral edges of the distal forelimb. A distinct interdigital notch forms between these developing digits. Corresponds with both Harrison and Glücksohn st. 41. 


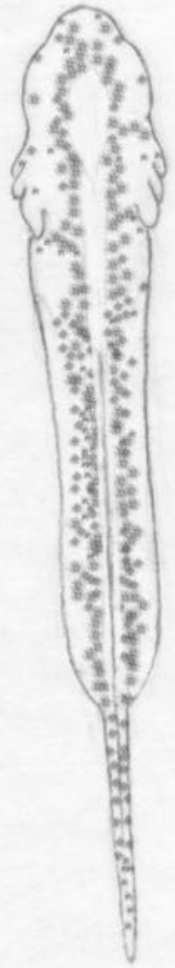

Limbfield-dorsal view (dors.)

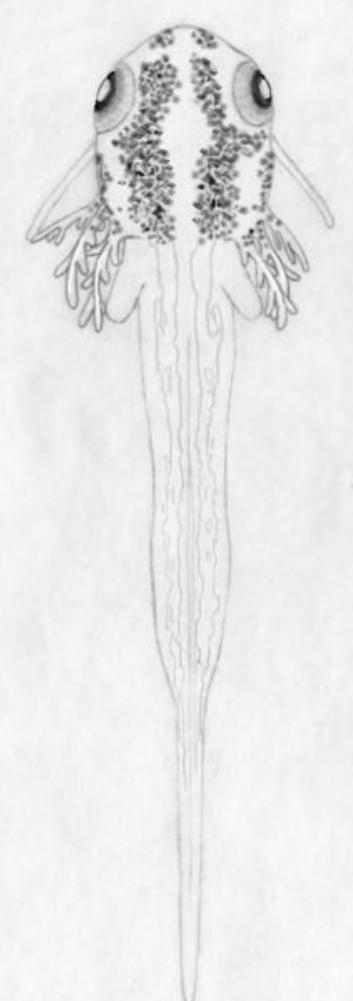

Peg stage-dors.

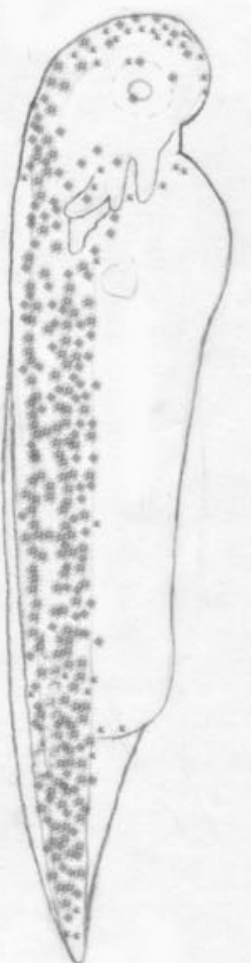

Limbfield-ventral view (vent.)

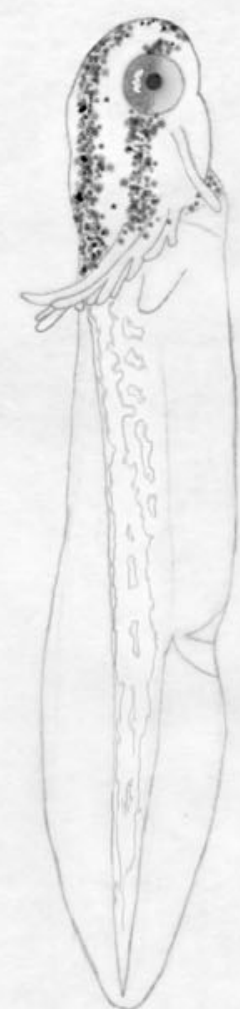

Peg stage-lat.

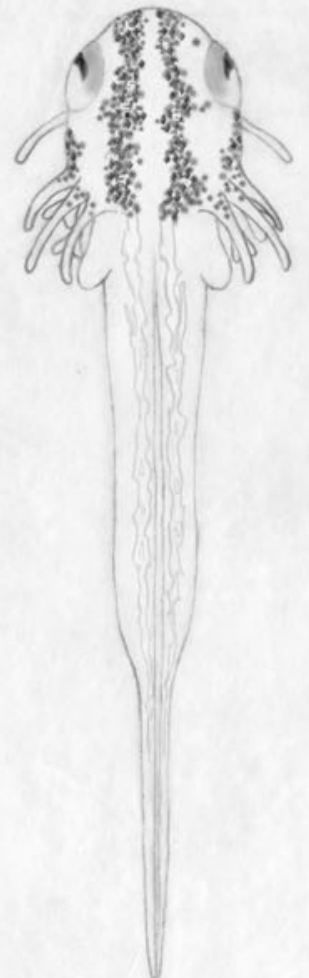

Bud stage-dors.

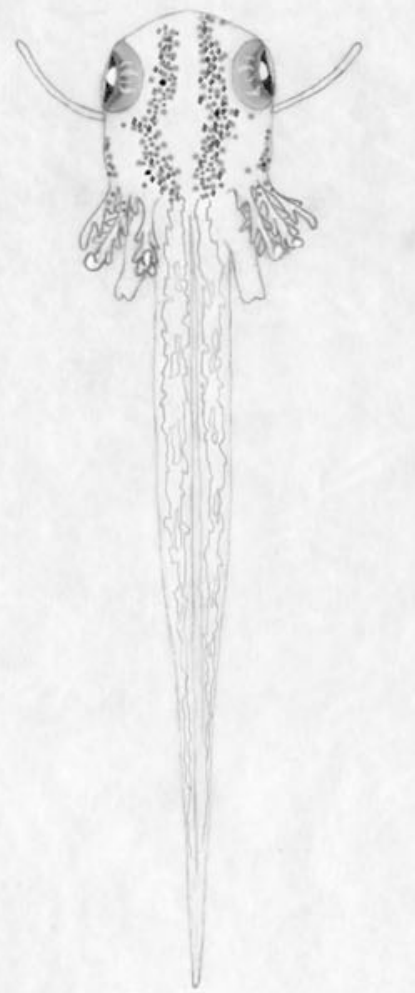

e2D stage-dors.

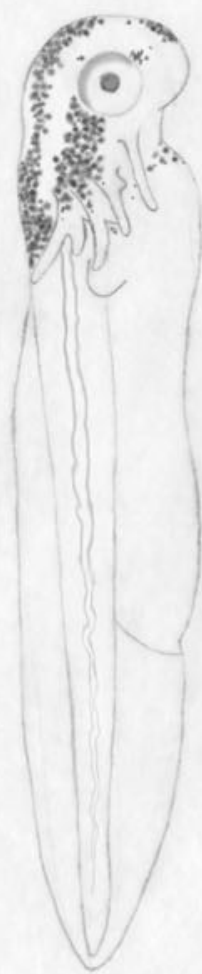

Bud stage-lateral view (lat.)

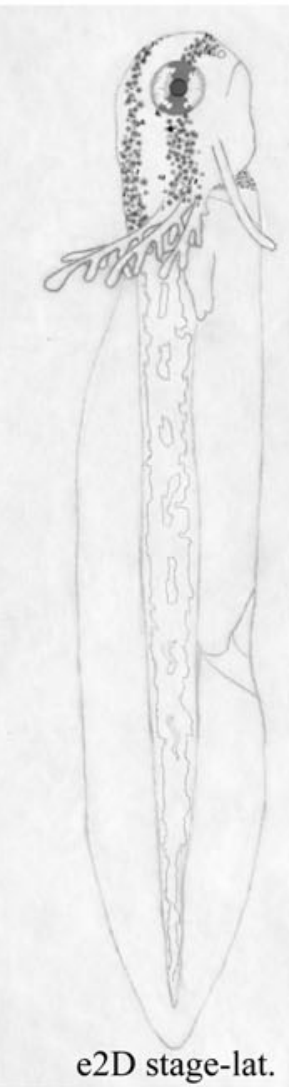




\section{Plate 2. Forelimb stages}

2D. Palm of the hand faces medially, open towards the body. Digit 1 is smaller than the more dorsal digit 2. Similar to both Harrison and Glücksohn st.43.

e3D. Entire hand is observed in dorsal view extending beyond the length of the external gills. Digit 2 is triangular in shape. Digit 1 used to anchor and balance the larva. A flattening along the dorsal edge of the forelimb at the base of digit 2 forms, from which digit 3 will develop. Cranial portion of the yolk mass begins to constrict.

3DI. Digit 3 emerges as a knob along the ulnar side of the forelimb. Limbs are held bent at the elbow. Larva begins to eat at this stage as the gut begins to form, with the cranial portion of the yolk mass taking on a tubular shape. Similar to both Harrison's and Glücksohn's st. 45 .

3DII. All three digits are digit-like in appearance. Entire gut is tubular within the peritoneal cavity, with the cranial portion beginning to twist. Larva begin to move head from side to side. Corresponds to Glücksohn st. 46 and similar to Harrison st. 46.

4D. Digit 4 emerges at this stage, first appearing as a small hillock adjacent to digit 3 and evolving into a knob. Forelimb is used in a coordinated walking motion. Eventually, D3>D2>D1>D4. Corresponds to Glücksohn st. 49. 


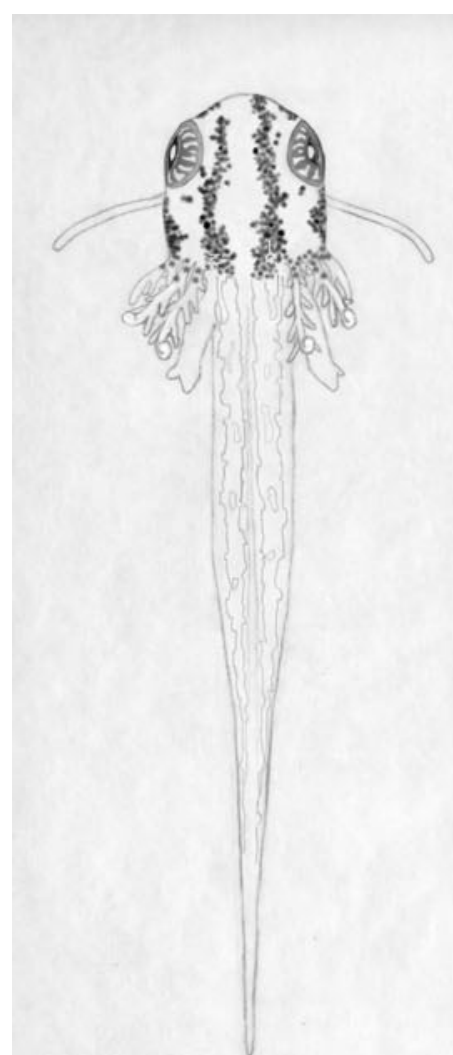

2D stage-dors.

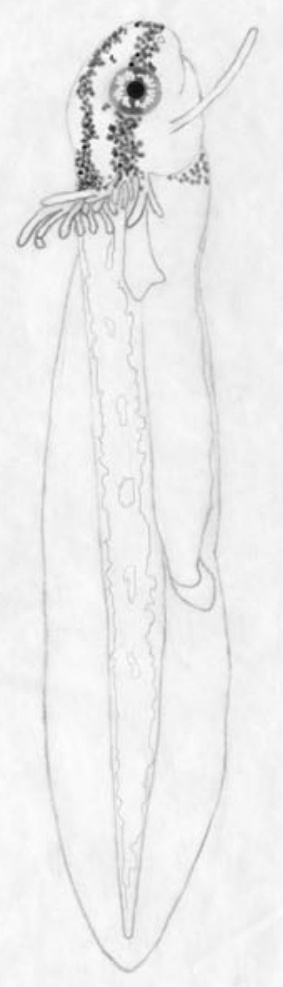

2D stage-lat.

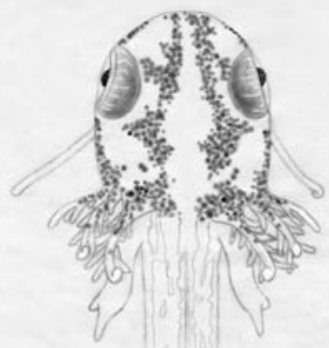

e3D stage-dors.

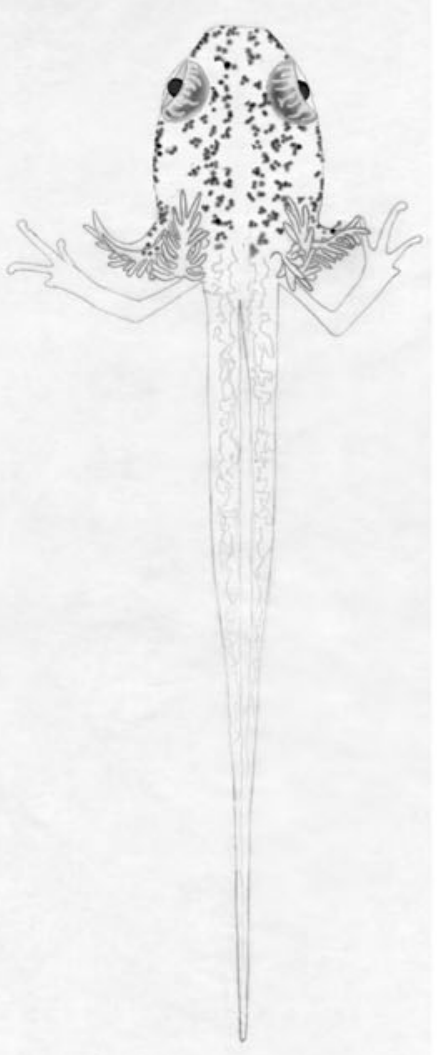

4D stage-dors.
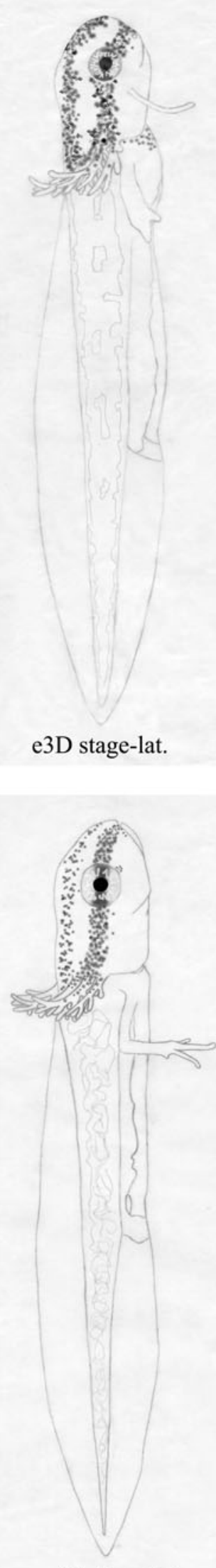

3DI stage-dors.

3DII stage-dors. 


\section{Plate 3. Hindlimb stages}

Limb field. Forms anywhere between 3DII and 4D stages. Small bilateral patches of thickened skin are visible ventrally, slightly cranial and lateral to the cloaca (indicated by the arrow).

Bud I. Limbs appear as small mounds at right angles to the ventral body wall, cranial and lateral to the cloaca (indicated by the arrow).

Bud II. The preaxial length increases prior to the development of the postaxial length, resulting in an 'L'-shaped hindlimb in ventral view. Similar to Harrison st. 45.

Bud III. Formation of a postaxial edge with the outgrowth of the limb. The distal tip is dome-shaped and can often be seen in dorsal view.

eT. Elongation of the preaxial edge beyond the postaxial edge results in an angular flattening of the distal tip in advance of early (e) toe formation. The appendage extends beyond the cloacal aperture. The distal portion flattens slightly in ventral view. Dorsally, it appears as a thin ridge running parallel to the body axis.

2T. First two toes appear concomitantly, with the distal tip forming toe two. The dorsal edge of the limb forms a thickened 'plate' from which toes 3, 4 and 5 will develop. Similar to Glücksohn st. 54. 


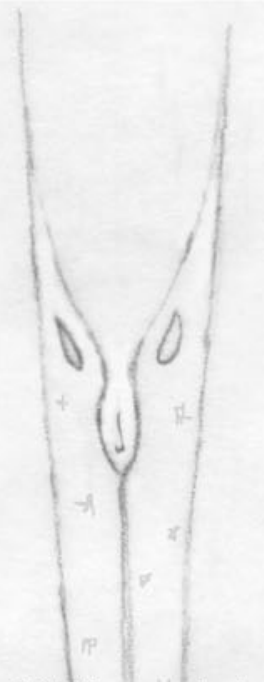

Limbfield-ventral view (vent.)

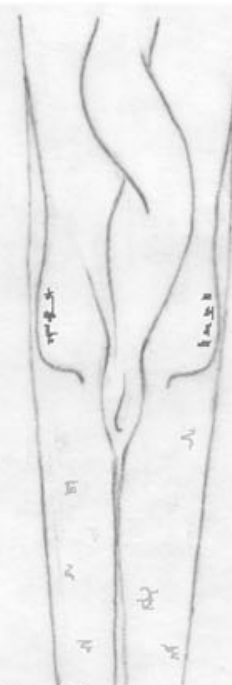

Bud II stage-vent.

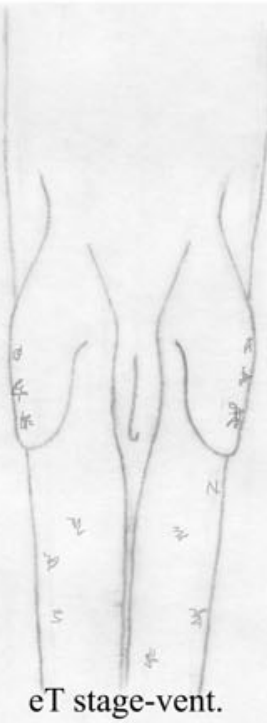

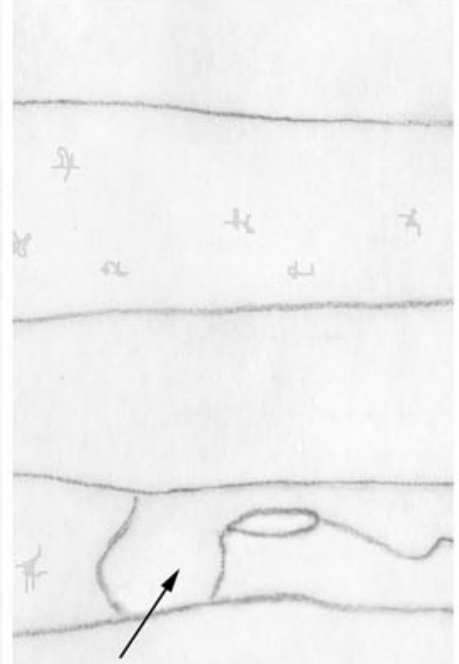

Limbfield-lat.

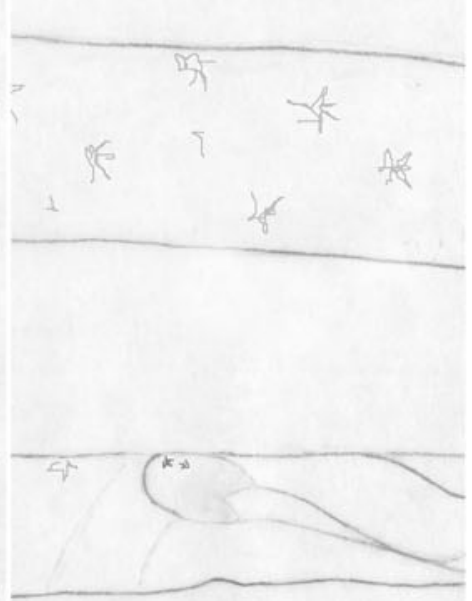

Bud II stage-lat.

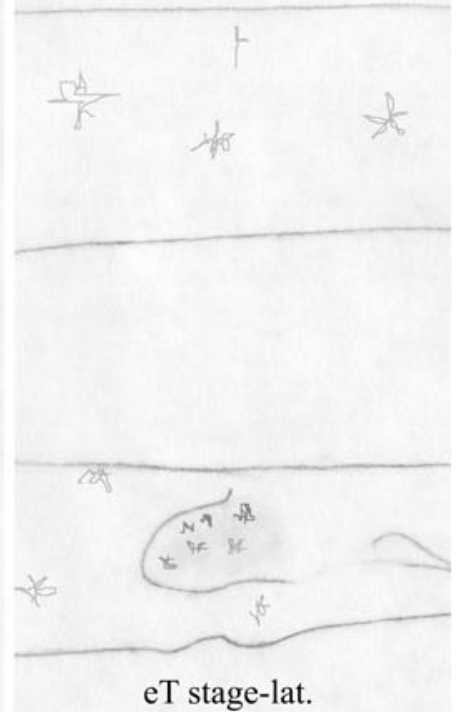

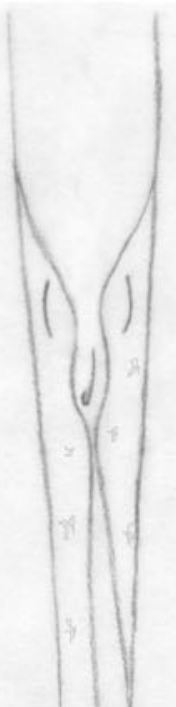

Bud I stage-vent.

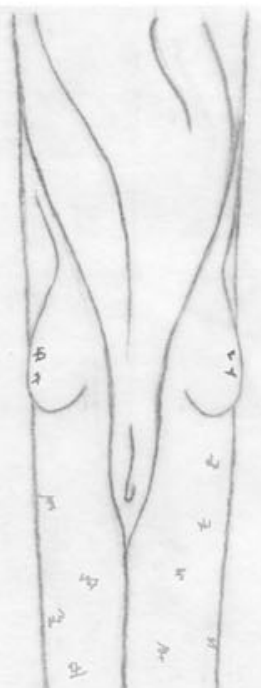

Bud III stage-vent.

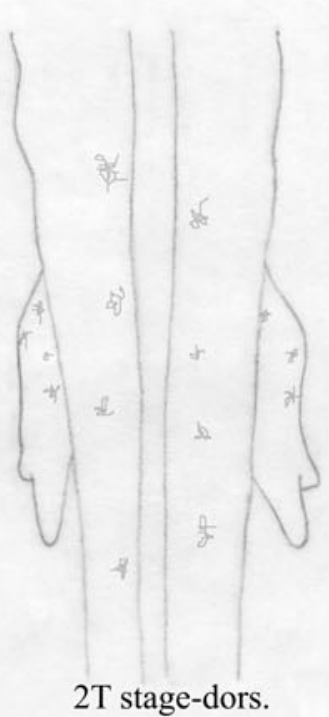

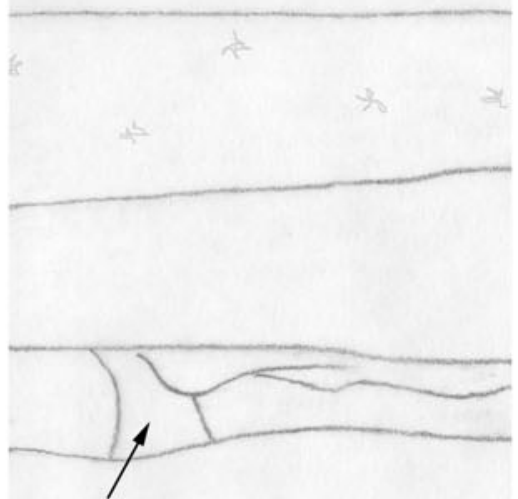

Bud I stage-lat.
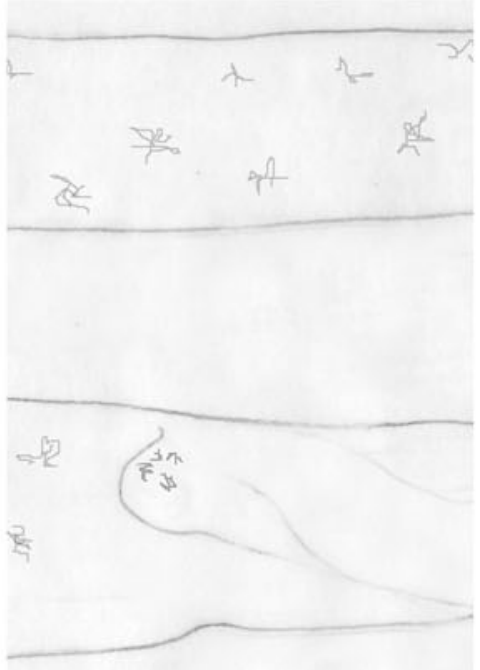

Bud III stage-lat.
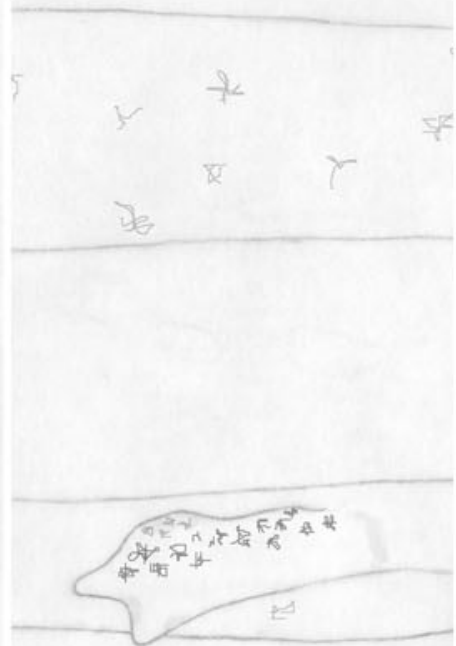

2T stage-lat. 


\section{Plate 4. Hindlimb stages}

3T. Toe 3 initially forms as a knob along the dorsal edge of the foot, to become triangular in shape. Toes 1 and 2 elongate. A distinct knee joint forms. A thickened 'plate' remains on the dorsal part of the limb from which toes 4 and 5 will form. Spasmodic movement of the limb occurs. Similar to Glücksohn st. 55.

4T. Toe 4 emerges as a mound on the dorsal hindlimb, adjacent to toe 3 , to form a knob and aligning with the other toes on the foot before it elongates. Toe 3 approaches the length of toe 2 and surpasses the extent of toe 1 . The knee is used in a walking motion. Similar to Glücksohn st. 57.

5T. Toe 5 emerges as a mound adjacent to toe 4, to grow into a knob. Eventually, T3>T4>T2>T1>T5. Similar to Glücksohn st. 59. 


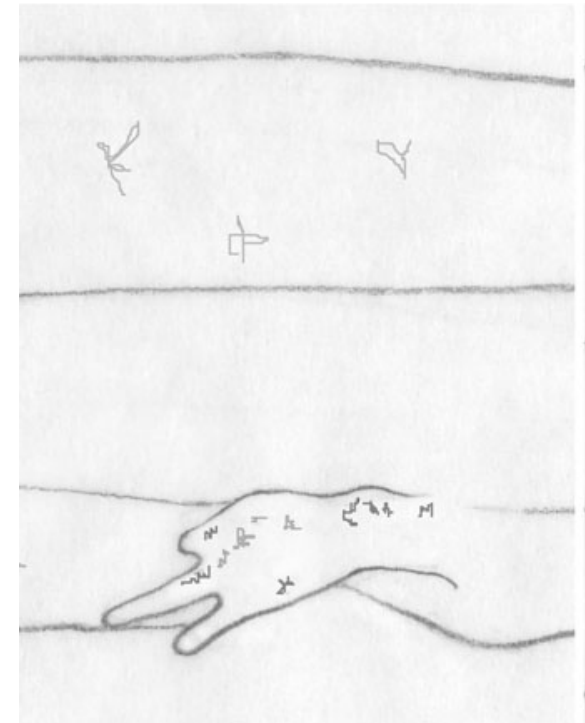

3T stage-lat.
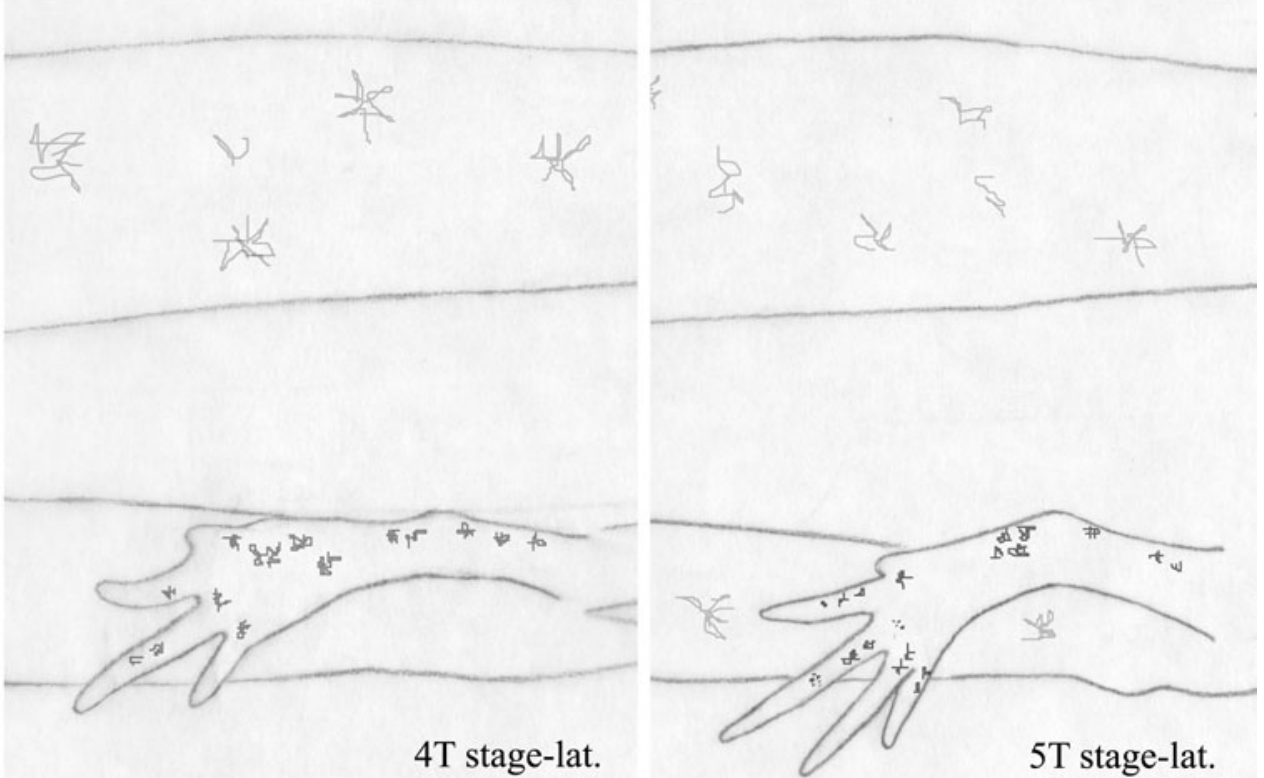

4T stage-lat.

5T stage-lat. 